\title{
Defining an Essential Clinical Dataset for Admission Patient History to Reduce Nursing Documentation Burden
}

\author{
Darinda E. Sutton ${ }^{1}$ Jennifer R. Fogel ${ }^{2}$ April S. Giard ${ }^{3}$ \\ Amy M. Rosa ${ }^{6}$ \\ ${ }^{1}$ Client Relationships: Clinical Leadership Team, Cerner Corp, Kansas \\ City, Missouri, United States \\ 2 Department of Clinical Informatics, Northern Light Health, Brewer, \\ Maine, United States \\ ${ }^{3}$ Department of Information Services, Northern Light Health, Brewer, \\ Maine, United States \\ ${ }^{4}$ Strategic Growth Organization: Hospital System Operations, Cerner \\ Corp, Kansas City, Missouri, United States \\ ${ }^{5}$ Department of Evidence Based Practice and Research, Vanderbilt \\ University Medical Center, Nashville, Tennessee, United States \\ ${ }^{6}$ Department of Nursing, SCL Health, Broomfield, Colorado, United States
}

Lisa A. Gulker ${ }^{4}$ Catherine H. Ivory ${ }^{5}$

Appl Clin Inform 2020;11:464-473.
Address for correspondence Darinda E. Sutton, MSN, RN-BC, FACHE, Cerner Corporation, 2800 RockCreek Parkway, Kansas City, MO 64117, United States (e-mail: darinda.sutton@cerner.com).

\section{Abstract \\ Keywords \\ - electronic health records \\ - documentation burden \\ - admission history \\ - registered nurse \\ - health information technology \\ - inpatient admission \\ - clinical care \\ - clinical informatics \\ - documentation elements}

Background Documentation burden, defined as the need to complete unnecessary documentation elements in the electronic health record (EHR), is significant for nurses and contributes to decreased time with patients as well as burnout. Burden increases when new documentation elements are added, but unnecessary elements are not systematically identified and removed.

Objectives Reducing the burden of nursing documentation during the inpatient admission process was a key objective for a group of nurse experts who collaboratively identified essential clinical data elements to be documented by nurses in the EHR.

Methods Twelve health care organizations used a data-driven process to evaluate inpatient admission assessment data elements to identify which elements were consistently deemed essential to patient care. Processes used for the twelve organizations to reach consensus included identifying: (1) data elements that were truly essential, (2) which data elements were explicitly required during the admission process, and (3) data elements that must be documented by a registered nurse (RN). Result The result was an Admission Patient History Essential Clinical Dataset (APH ECD) that reduced the amount of admission documentation content by an average of $48.5 \%$. Early adopters experienced an average reduction of more than two minutes per admission history documentation session and an average reduction in clicks of more than $30 \%$.

Conclusion The creation of the essential clinical dataset is an example of combining evidence from nursing practice within the EHR with a set of predefined guiding principles to decrease documentation burden for nurses. Establishing essential documentation components for the adult admission history and intake process ensures the efficient use of bedside nurses' time by collecting the right (necessary) information collected by the right person at the right time during the patient's hospital stay. Determining essential elements also provides a framework for mapping components to national standards to facilitate shareable and comparable nursing data. received

November 21, 2019

accepted after revision

May 18, 2020 (c) 2020 Georg Thieme Verlag KG Stuttgart · New York
DOI https://doi.org/ $10.1055 / \mathrm{s}-0040-1713634$. ISSN 1869-0327. 


\section{Background}

Documentation burden is significant for nurses and contributes to decreased time with patients as well as clinician burnout. ${ }^{1}$ A recent systematic review suggests that documentation efficiencies are gained in the first weeks after health care IT(HIT) implementation, but documentation burden increases with the longer time HIT systems are in place. ${ }^{1}$ A contributor to documentation burden over time may be that while new documentation requirements are frequently added, unnecessary elements are rarely systematically identified and removed. ${ }^{1}$ Further, as the profession closest to patients, nurses may be perceived as the logical user group to which documentation requirements are added. Rarely is an analysis conducted to determine whether documentation could be accomplished by another member of the health care team, leaving more time for nurses to spend providing patient care. The acute care admission process is a significant event in the patient care episode. Specific documentation is necessary to plan and execute nursing care, but too much documentation does not add value to the care team.

This article describes a process for simplifying documentation requirements during the acute care inpatient admission process by creating a minimally required dataset. The process is vendor-neutral and can be applied across settings. The resulting dataset was tested for feasibility by early adopter organizations. In addition to describing the process to create the minimized dataset, we report pre- and postimplementation metrics for early adopters and provide suggested best practices for simplifying datasets used in other aspects of clinical care.

\section{Significance of the Problem}

Nurses are the largest end-users of electronic documentation systems in health care. The burden of collecting data and information through nursing documentation takes time away from patient care and leads to nurse burnout. ${ }^{2}$ Literature suggests that nurses spend as much as $50 \%$ of their day performing administrative tasks, including interacting with the medical record. ${ }^{3}$ Redundant documentation and regulatory requirements contribute to documentation burden, ${ }^{4}$ defined as the completion of unnecessary documentation elements in the electronic health record (EHR). ${ }^{5}$ In an era where nurse leaders must demonstrate the value of nursing care to patient outcomes, it is more important than ever that data generated by nurses are useful in telling the patient story and highlight the unique contribution of nurses to that story. Clinical informatics teams representing clinical endusers and EHR vendors are required key partners with nursing operations leaders in reducing documentation burden to visualize the value of nursing care to patient outcomes.

National HIT organizations recommend the use of standard terminologies and datasets to facilitate HIT interoperability $^{6}$ and various terminologies exist that support nursing practice, but none have been widely deployed. ${ }^{7}$ To adopt HIT standards, nursing professionals must decide which data points are necessary for inclusion in the context of patient care, what documentation falls in the scope of nursing practice, and what required data could be documented by another member of the health care team. A large EHR vendor, in collaboration with informatics and nursing practice experts, created an essential clinical dataset of components required when nurses admit an adult patient in an acute care setting; the dataset is referred to as the Admission Patient History Essential Clinical Dataset (APH ECD).

\section{Objectives and Purpose Statement}

The primary purpose of the essential clinical dataset initiative was to systematically reduce the amount of patient data gathered and recorded by nurses during the inpatient acute care admission process, as well as increase nursing satisfaction due to a decrease in documentation burden. Aspects of the physical assessment were considered out of scope for this project. Physical assessment occurs at various times during an inpatient stay; therefore, physical assessment essential data are to be determined by a future work team. A secondary purpose of the initiative was to create and evaluate the feasibility of a dataset that can be operationalized across settings and mapped to existing terminology standards to facilitate shareable and comparable nursing documentation data.

\section{Project Setting}

The EHR vendor organized a diverse group of client organizations, who varied in hospital size, type (community or academic), and geographic location, referred to as an APH ECD Collaborative. The twelve APH ECD Collaborative organizations accounted for approximately 46,000 acute care beds and 192 hospitals in the United States, including academic health systems, integrated delivery networks (IDN), and one community hospital. Each organization identified a key decision-maker who was either a Chief Nursing Officer or a Chief Nursing Informatics Officer. Each collaborative organization formed an internal team to support the work, selecting participants as needed. Facility and system team members included bedside nurses, nursing analysts, and nursing informatics specialists. The APH ECD Collaborative was launched in June 2016 and met monthly for 2 years via virtual meetings, augmented by semiannual face to face meetings.

\section{Methods}

Nurses working at the top of their license while documenting patient care was a vital aspect of the methodology. As the American Nurses Association Principle for Nursing Documentation states, "nurses should aim to ensure that critical and necessary data and information are documented while avoiding duplicative documentation. To do so, nurses need to understand the forces and other factors that shape the requirements of practice-specific documentation." ${ }^{8}$ The collaborative determined that the data considered for inclusion must be essential 
for collection by the RN during the admission process to ensure alignment with this guiding principle.

\section{Development of an APH ECD}

Along with the guiding principles, the collaborative members considered three criteria to assess whether data elements were essential for nursing to document during the admission process. Data elements meeting any or all the following criterion were determined for the essential clinical dataset: (1) the data element was evidence-based, (2) the data element was nursing practice-based, meaning the majority of collaborative members documented the element during patient admission and (3) U.S. federal regulatory agencies required the data element.

As a first step, the collaborative members performed a literature search to review the evidence with terms such as "data elements required to be collected by nursing at the time of inpatient admission" and found no evidence to support specific requirements that RNs must collect any particular data at the time of admission intake history. Lack of evidence supported the notion that some data elements currently documented at admission could be documented at another time. The literature search pertained to admission history content only; evidence related to outcomes was out of scope for this collaborative. Although evidence was not found for specific content of inpatient adult admission history, the members of the collaborative felt that reviewing existing evidence should remain a part of the process in developing any essential clinical dataset.

Following the literature review, 3 months of admission history data were extracted from the EHR databases of the 12 collaborative organizations. A review of the list of admission history data elements provided evidence from nursing practice. The following steps were applied to compare the datasets to determine the frequency and utilization of the data elements in the admission history:

1. A side by side comparison of all 12 datasets was completed to determine which data elements were in common among them.

2. Frequency was defined as eight or more of the clients having the data element available for use in documentation.

3. Utilization was defined as the actual number of times the data element was documented.

4. From this comparison, the frequency that each data element appeared in common was calculated. For example, some data elements were in common among several datasets, and others were in common more frequently. Consensus for the data element was reached if data elements were present in eight or more datasets

5. Average utilization was calculated from the number of times the data element was documented, divided by the number of times the data element was available.

\section{Reviewing Regulatory Requirements}

The members of the collaborative engaged an objective, external consulting company with expertise in the standards for the following regulatory agencies: The Joint Commission (TJC), Det Norske Veritas, and Centers for Medicare and
Medicaid Services. The members of the collaborative participated in an 8-hour in-service with the consultants related to the standards for adult inpatient admission history information. The collaborative members then evaluated the dataset against the standards criteria. The collaborative confirmed 27 of the data elements applied to the standards that must be collected at admission. The collaborative then shared the findings with the external consultants, who, in turn, validated that the assessment of those 27 data elements was appropriate.

\section{Determination of the Essential Clinical Dataset}

By completing the entire process described above, the collaborative created an APH ECD that was evidence, regulatory, and practice based. -Table 1 identifies the data elements deemed essential for intake during the admission process.

\section{Feasibility Evaluation}

Early-adopter organizations evaluated the feasibility of the APH ECD by measuring quantitative outcomes. Metrics associated with the amount of time nurses spent documenting the admission history were assessed using EHR system timers and event logs. The EHR system logs also provided the total number of clicks expended during the completion of the new documentation form. Outcomes included pre- and 30 days post-implementation measures of:

- The number of required data elements documented. Distinct data elements on the acute inpatient admission history electronic form(s) were compared between baseline and post-implementation of the APH ECD.

1. The time required to complete admission documentation. The EHR system timers calculated the total time to complete a new documentation form in minutes and seconds.

2. The number of required clicks. The EHR event logs summarized the total number of clicks expended during the completion of the new documentation form.

If the total amount of documentation content was reduced, the collaborative members expected to see a reduction in both the amount of time and number of clicks required to complete a documentation session for an adult acute inpatient admission history, reducing documentation burden.

\section{Results}

\section{Documentation Content}

The findings in the number of data elements across the group of early adopters ranged from an increase of $5.1 \%$ of data elements to a reduction of $90.9 \%$ data elements, with an average reduction in data elements for the entire cohort of 48.5\%; findings are illustrated in - Fig. 1 .

\section{Documentation Time}

-Fig. 2 illustrates the average time to complete a documentation session across ten organizations 30 days after the implementation of the new APH ECD content. The early 
Table 1 Essential clinical dataset elements

\begin{tabular}{|c|c|c|c|c|c|}
\hline & Section label & Data element & $\begin{array}{l}\text { Evidence - } \\
\text { Based } \\
\text { Practice }\end{array}$ & Regulatory & $\begin{array}{l}\text { Evidence } \\
\text { from } \\
\text { nursing } \\
\text { Practice }\end{array}$ \\
\hline \multirow[t]{2}{*}{1} & General information & Preferred spoken language & & $x$ & \\
\hline & & $\begin{array}{l}\text { These data elements only apply if Spoken Language is } \\
\text { other than English }\end{array}$ & & & \\
\hline $1 \mathrm{a}$ & Interpretive services & Interpretation modality & & $x$ & \\
\hline $1 b$ & Interpretive services & Interpreter services declined reason & & $x$ & \\
\hline $1 \mathrm{c}$ & Interpretive services & Interpreter staff/in-house & & $x$ & \\
\hline $1 d$ & Interpretive services & Interpreter name & & $x$ & \\
\hline 1e & Interpretive services & Interpreter ID number & & $x$ & \\
\hline $1 f$ & Interpretive services & Interpreter agency name & & $x$ & \\
\hline 2 & General information & Preferred written language & & $x$ & \\
\hline 3 & General information & Preferred mode of communication & & $x$ & \\
\hline 4 & General information & Sensory deficits & & & $x$ \\
\hline 5 & General information & Sensory devices needed & & & $x$ \\
\hline 6 & General information & Sensory items at bedside & & & $x$ \\
\hline 7 & General information & Preferred name & & & $x$ \\
\hline 8 & General information & Admission information given by & & $x$ & \\
\hline 9 & General information & Pregnancy status (only for female patients) & & & $x$ \\
\hline 10 & Advance directives & Advance directive & & $x$ & \\
\hline 11 & Advance directives & $\begin{array}{l}\text { Patient wishes to receive further information on } \\
\text { advance directives }\end{array}$ & & $x$ & \\
\hline 12 & Infectious disease risk screening & Recent travel history (Patient) & & & $x$ \\
\hline 13 & Infectious disease risk screening & Recent travel location (Patient) & & & $x$ \\
\hline 14 & Infectious disease risk screening & Family member travel history & & & $x$ \\
\hline 15 & Infectious disease risk screening & Family member recent travel location & & & $x$ \\
\hline 16 & Infectious disease risk screening & Infectious disease symptoms-20 $\mathrm{Y} / \mathrm{N}$ answers & & & $x$ \\
\hline 17 & Infectious disease risk screening & Tuberculosis risk factors $-8 \mathrm{Y} / \mathrm{N}$ answers & & & $x$ \\
\hline 18 & Infectious disease risk screening & Tuberculosis description & & & $x$ \\
\hline 19 & Infectious disease risk screening & Date of treatment for TB & & & $x$ \\
\hline 20 & Infectious disease risk screening & Verify droplet, contact isolation for Ebola & & & $x$ \\
\hline 21 & Infectious disease risk screening & Verify airborne, contact isolation for MERS & & & $x$ \\
\hline 22 & Infectious disease risk screening & $\begin{array}{l}\text { Condition compromising immune system-22 } \\
\text { selections }\end{array}$ & & & $x$ \\
\hline 23 & Nutritional screening & Home diet & & $x$ & \\
\hline 24 & Nutritional screening & Nutritional risk factors & $x$ & $x$ & \\
\hline 25 & $\begin{array}{l}\text { Columbia Suicide Severity Rating } \\
\text { Scale }^{9}\end{array}$ & $\begin{array}{l}\text { Have you wished you were dead or wished you } \\
\text { could go to sleep and not wake up? }\end{array}$ & $x$ & $x$ & \\
\hline 26 & $\begin{array}{l}\text { Columbia Suicide Severity Rating } \\
\text { Scale }^{9}\end{array}$ & $\begin{array}{l}\text { Since last asked, have you actually had any thoughts } \\
\text { of killing yourself? }\end{array}$ & $x$ & $x$ & \\
\hline 27 & $\begin{array}{l}\text { Columbia Suicide Severity Rating } \\
\text { Scale }^{9}\end{array}$ & $\begin{array}{l}\text { Since last asked, have you been thinking about how } \\
\text { you might kill yourself? }\end{array}$ & $x$ & $x$ & \\
\hline 28 & $\begin{array}{l}\text { Columbia Suicide Severity Rating } \\
\text { Scale }^{9}\end{array}$ & $\begin{array}{l}\text { Since last asked, have you had these thoughts and } \\
\text { had some intention of acting on them? }\end{array}$ & $x$ & $x$ & \\
\hline 29 & $\begin{array}{l}\text { Columbia Suicide Severity Rating } \\
\text { Scale }^{9}\end{array}$ & $\begin{array}{l}\text { Since last asked, have you started to work out or } \\
\text { worked out the details of how to kill yourself? Do } \\
\text { you intend to carry out this plan? }\end{array}$ & $x$ & $x$ & \\
\hline 30 & $\begin{array}{l}\text { Columbia Suicide Severity Rating } \\
\text { Scale }^{9}\end{array}$ & $\begin{array}{l}\text { Since last asked, have you ever done anything, } \\
\text { started to do anything, or prepared to do anything } \\
\text { to end your life? }\end{array}$ & $x$ & $x$ & \\
\hline 31 & Educational needs/learning style & Barriers to learning & & $\mathrm{x}$ & \\
\hline
\end{tabular}


Table 1 (Continued)

\begin{tabular}{|l|l|l|l|l|}
\hline & Section label & Data element & $\begin{array}{l}\text { Evidence - } \\
\text { Based } \\
\text { Practice }\end{array}$ & $\begin{array}{l}\text { Regulatory } \\
\text { Evidence } \\
\text { from } \\
\text { nursing } \\
\text { Practice }\end{array}$ \\
\hline 32 & Educational needs/learning style & Patient learning style preference & & $\mathrm{X}$ \\
\hline 33 & Visitors & $\begin{array}{l}\text { Will anyone be staying with you while in the } \\
\text { hospital? }\end{array}$ & & $\mathrm{X}$ \\
\hline 34 & Visitors & Relationship to patient & & $\mathrm{X}$ \\
\hline 35 & Visitors & Visitor Name & & $\mathrm{X}$ \\
\hline 36 & Visitors & Visitor restrictions (Y/N) & & $\mathrm{X}$ \\
\hline 37 & Visitors & free-text comment & & $\mathrm{X}$ \\
\hline 38 & Anticipated discharge needs & Discharge to & & \\
\hline Additional elements that are part of the longitudinal patient record and validated/captured by the RN as part of the Admission Intake process & $\mathrm{X}$ \\
\hline & Social history & $\begin{array}{l}\text { Tobacco, alcohol, substance use, abuse and ne- } \\
\text { glect, nutrition and diet }\end{array}$ & & \\
\hline & Allergies & Allergies & & $\mathrm{X}$ \\
\hline & Home medications & Home medications and compliance & $\mathrm{X}$ \\
\hline & Immunizations & Influenza & $\mathrm{X}$ & \\
\hline
\end{tabular}

Abbreviations: MERS, Middle East respiratory syndrome; TB, tuberculosis.

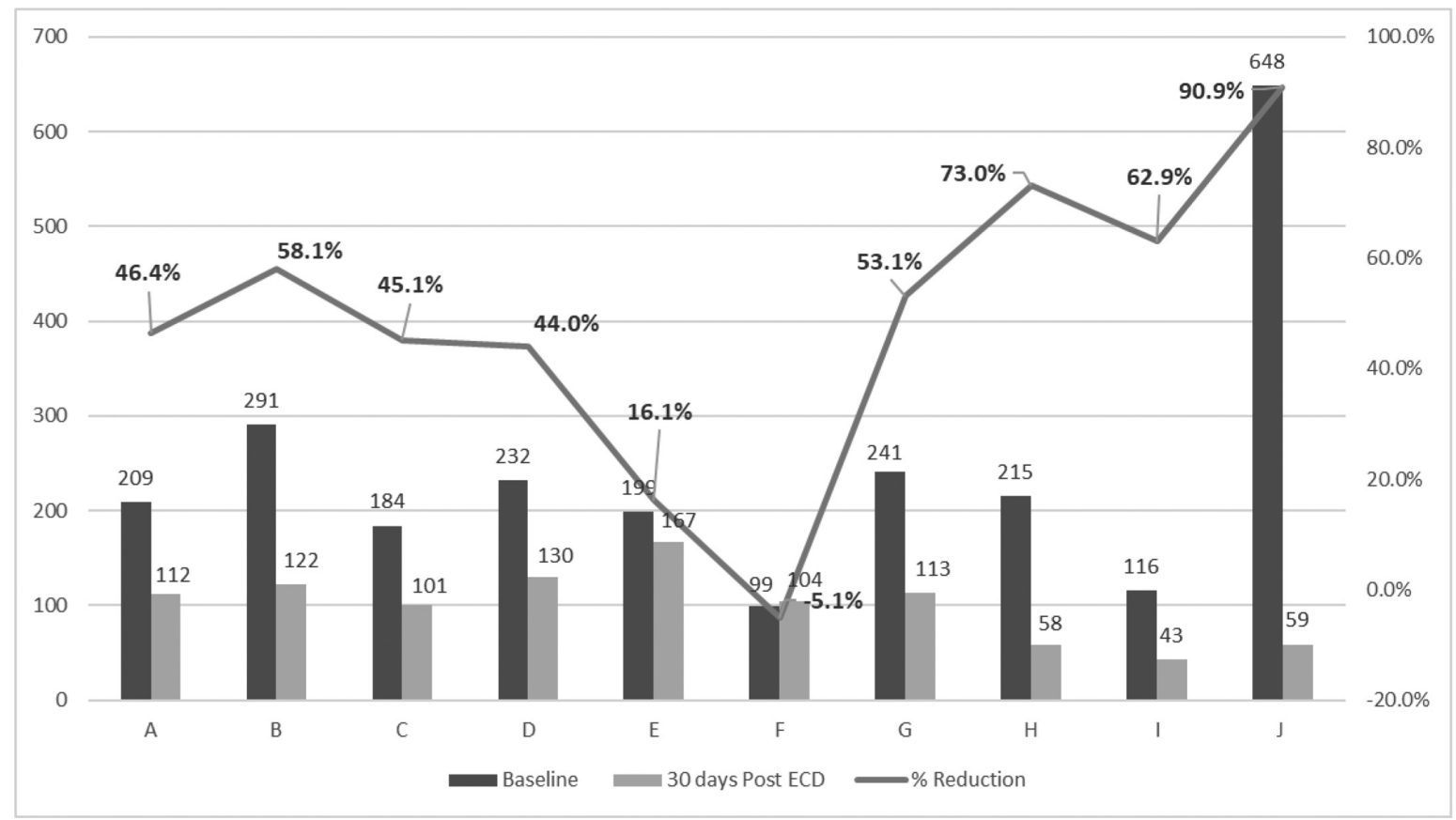

Fig. 1 Total data elements and percent reduction adult acute inpatient admission history baseline versus 30 days post APH ECD. APH ECD, Admission Patient History Essential Clinical Dataset.

adopter organizations showed an average reduction of 2 minutes and 2 seconds, or a $30.6 \%$ reduction, in time spent completing one documentation session.

\section{Documentation Clicks}

-Fig. 3 illustrates the total number of clicks, with an average percent change, to complete the admission history intake across ten organizations from baseline to 1-month postimplementation of the new APH ECD content. The average change was a reduction of $32 \%$ or 37 actual clicks. The range for the percent change in the number of clicks in a documentation session measured from +19 to $-76 \%$.

\section{Discussion}

Based on our evaluation, the use of the APH ECD is feasible. Documentation burden was reduced for most users based on decreased content, time, and the number of clicks. Documentation content decreased by an average of $48.5 \%$. Time reduced by an average of $30.6 \%$, and the number of clicks 


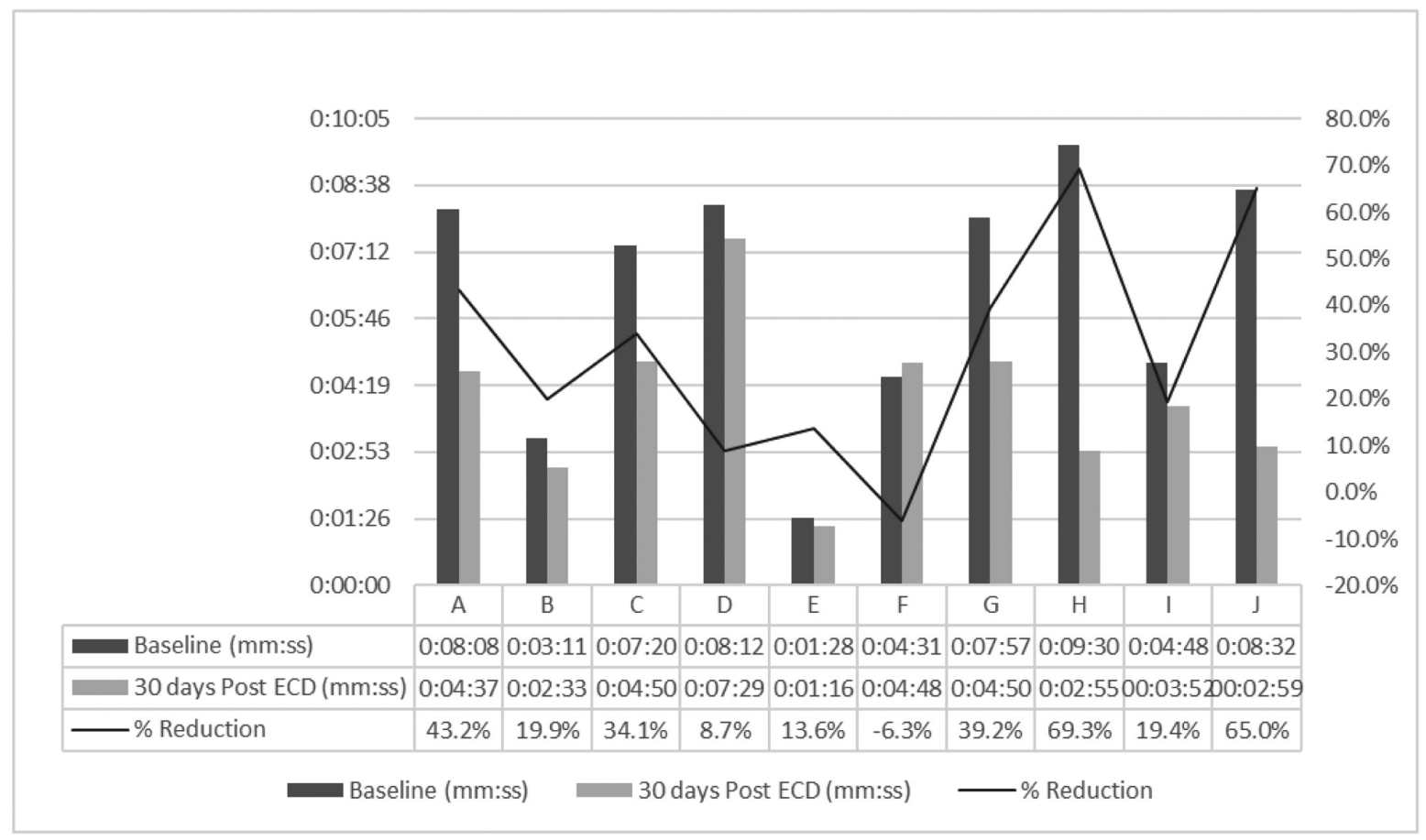

Fig. 2 Average total time to document: adult acute inpatient admission history baseline versus 30 days post APH ECD. APH ECD, Admission Patient History Essential Clinical Dataset.

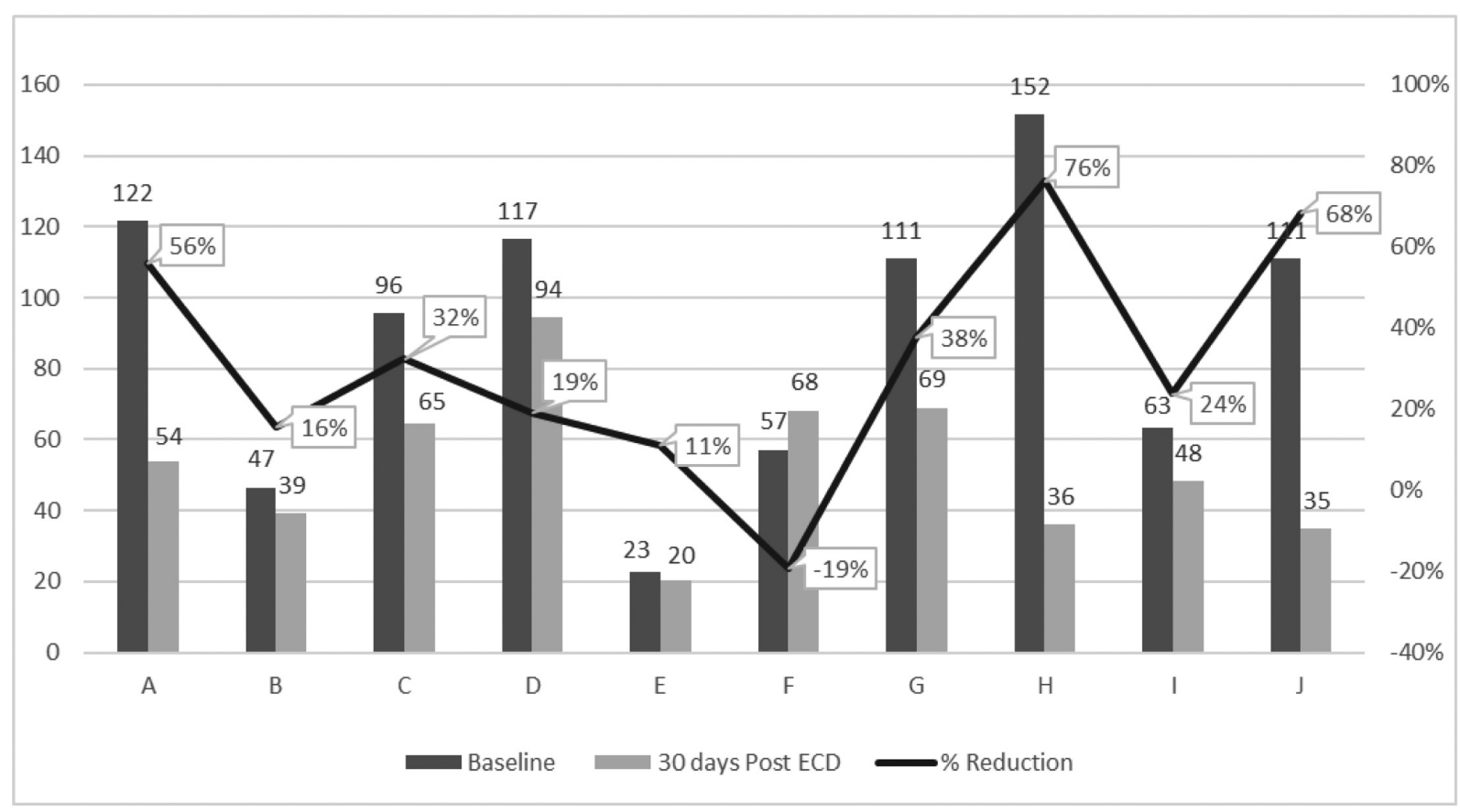

Fig. 3 Average total clicks to document: adult acute inpatient admission history baseline versus 30 days post APH ECD. APH ECD, Admission Patient History Essential Clinical Dataset.

reduced by $32 \%$. Past informatics research and quality studies support the use of EHR system timers and event logs to measure the pre- and post-intervention changes in efficiency and quality of a defined clinical workflow into an EHR. ${ }^{10-12}$

Organization F, a large Integrated Delivery Network (IDN), actually experienced an increase in the number of data elements, time per documentation session, and clicks per documentation session after implementing the APH ECD content (five element increase (5.1\%); 17-second increase, (6.3\%); 11 click increase, (19.2\%). While content was removed from the adult acute inpatient admission intake, a social history tool for Meaningful Use 3 reporting for sexual orientation and gender identity was added to the inpatient documentation workflow. Clinical leadership at Organization $\mathrm{F}$ believes that over time documentation efficiency will increase because the social history documentation will carry 
across patient encounters as clinicians in various venues contribute to the longitudinal record. The feasibility evaluation also identified best practices for implementing the APH ECD.

Collaborative members recognized that end-user input is a valuable step when adopting change, especially as it relates to the EHR. As such, one early adopter of the APH ECD used a shared governance model to solicit feedback on the proposed changes to current data captured on the admission history. This approach leveraged a standardized, repeatable process that did not require a formal setting to gather feedback, yet still provided a clear understanding of the proposed changes.

The feasibility assessment process used by early adopter organizations to implement the APH ECD provided a format that can be replicated by anyone adopting the APH ECD. The process is agnostic of the EHR vendor. Best practices for implementing a standard dataset include engaging staff from the clinical informatics department to complete a crosswalk of the APH ECD with the facility's current admission history and intake form, reviewing each data element for alignment with the APH ECD, and considering information that is required to meet state regulations or local sentinel event action plans. Additional best practices include:

1. Validate that physical assessment data removed from the admission history and intake form is available for documentation within the physical assessment documentation section.

2. Convert the crosswalk into a presentation (recommend PowerPoint as this format can be presented to various sized audiences).

3. Explain the "why" (decreasing documentation burden and increasing workflow efficiencies during the admission history and intake process).

4. Outline the steps required for the review to ensure a consistent process.

5. Remind staff to "think outside the box" and ask themselves if the data elements:

a. must be collected by an RN.

b. Are essential regardless of what current hospital policy might currently dictate (unless it is a regulatory requirement).

c. if necessary, is the admission process the correct workflow to gather the information,

6 . Create screenshots of each section of the current admission history and intake form.

7. Using icons, designate the element with a:

a. Green Checkmark-KEEP: corresponds to the APH ECD or is essential for the state, local, or sentinel event tracking.

b. Red X-REMOVE: is collected by another health care provider or provides no value to the care of the patient.

c. Purple Arrow-MOVE: to another more appropriate section of the EHR.

d. Yellow Question Mark-QUESTION on workflow or a process review required before making the final decision.
8. Evaluate the downstream impact on clinical decision support rules, reports, and additional secondary use of the elements proposed for removal.

9. Identify and prepare champions who will share the documentation changes and gather feedback from bedside nurses who complete the admission history and intake form.

10. Establish a timeframe for the review process that allows the champion to speak with nurses from various shifts and different levels of experience.

11. If any nurse disagrees with a decision, ask for the rationale on why this information is essential to admission history and intake process.

12. Following the review process, meet with the champions to discuss the comments and feedback, taking any recommendation for changes to the proposed model back to the governance committee for final approval.

The review process represented a shared governance approach that allowed staff to contribute to the design and build (where and how to place data elements) to decrease documentation burden. Critical to the review process was the need to challenge the nurse's traditional thinking on admission documentation requirements. For example, when staff questioned the various elements identified for removal, they were asked to explain why it was crucial to the care of the patient at the time of admission as well as the benefit of keeping the data as an essential element. Often, no clear justification other than "nurses have always documented this" could be provided. Once the change process was complete, nurses voiced excitement about the changes, appreciative of the time savings, and the elimination of duplicate documentation. The early adopter organization indicated that this response was very different from prior EHR changes when documentation elements were changed and or increased in number rather than focused on the elimination of documentation burden.

Interestingly, nursing leadership was more skeptical of the changes than the bedside nurse. Concerns were voiced that crucial elements of documentation needed to meet regulatory requirements would be missing. However, a post-implementation review of the documentation demonstrated no untoward effects or missing documentation. Since the initial implementation of the APH ECD, one early adopter organization has experienced two accreditation visits by TJC with no citations nor recommendations for improvement received.

The inpatient, medical-surgical adult population was the first specialty area selected to utilize the process of defining an APH ECD for admission. New ECD collaboratives have been organized to focus on pediatric patient admission and physical assessment, abbreviated adult admissions, such as 23-hour observation and preoperative or preprocedural admissions. The capture of data elements that are unique to a nursing specialty is deemed important. As an example, specialty ECD collaboratives for behavioral health and obstetric populations' intake admission have been initiated. The adult critical care physical assessment is another venue of care where work has begun to define the ECD. Additionally, this process of generating 
evidence from nursing-practice may be utilized by other EHR cohorts, inclusive of those outside of the U.S., as global professional nursing associations are publishing position statements regarding documentation. The Canadian Nurses Association and the Canadian Nursing Informatics Association published a joint position statement in March 2017 recognizing the need for "a standardized approach to nursing documentation in all clinical practice settings across Canada." ${ }^{13}$ Australia unveiled the Nursing Informatics Position Paper on July 10, 2018, produced collaboratively by the Australian College of Nursing, Health Informatics Society of Australia, and its special interest group Nursing Informatics Australia. Element 7 states: "Nurse informaticians insist on the adoption of nationally agreed nursing data standards, data quality standards, and interoperability that allow the exchange of clinical information meaningfully across healthcare systems for improved data integration, information sharing, performance monitoring, data analytics, patient safety and quality."14

\section{Study Limitation}

The authors acknowledge that a convenience sample of organizations aligned with a single EHR vendor may be a limitation of the APH ECD. However, the process can be replicated and is vendor-neutral in its approach. Further, this project focused only on data elements collected by nursing at a particular time during a patient's hospital stay.

\section{Implications for Clinical Informatics Practice}

Changes to documentation within the EHR have implications for accuracy, efficiency, user satisfaction, and workflow. ${ }^{15}$ Clinicians want assurance that technology supports and enables the care they provide instead of driving or defining their practice. Nurse leaders need assurance that time spent with technology, including the EHR, does not take the bedside nurse away from the patient any more than is necessary. The work completed by the APH ECD collaborative demonstrates a commitment to the delivery of high quality, safe patient care by decreasing documentation burden. Additionally, by using a clinically driven and data-driven process that leverages a shared governance structure of decision making, future requests for changes to the EHR will utilize these ECD concepts as the foundation for change control processes. The work is also an example of generating practice-based evidence from the EHR, complimenting work published by Embi and Payne that recognized the benefits of leveraging data collected through the delivery of health care to knowledge gathering processes. ${ }^{16}$ Establishing the essential documentation components for the adult admission history and intake process ensures the efficient use of bedside nurses' time by collecting the right essential information, collected by the right person (the professional RN) at the right time during the patient's hospital stay. All these aspects contribute to the decrease in the burden of documentation.

This work lays the foundation for a repeatable process for defining data elements that are essential for documentation in other venues of care and other clinical documentation workflows. The APH ECD is EHR vendor-independent in its approach and applicable across all EHR platforms for defining the essential content. Due to its universal applicability, the APH ECD can serve as a foundation for not only U.S. standardization, but global and international efforts that are seeking to define a standard across a country or region.

\section{Adopting APH ECD As the Standard}

Now that the APH ECD has been defined and adopted in clinical settings across a broad set of clients and EHR platforms, we propose its adoption as the industry standard dataset that is mappable to Logical Observation Identifiers Names and Codes (LOINC) and SNOMED-CT terminologies/concepts. Once standards like the APH ECD are adopted and recognized, our profession can share data and begin to compare nursing practice, patient care outcomes, and clinical interventions across large national and international populations. Once the LOINC and SNOMED-CT mapping are complete, an analysis can be initiated to determine if additional data elements are present in these terminologies that should be included in the APH ECD. Even when adopted as a national standard, each organization will have to localize the APH ECD, or any dataset, to meet state and local requirements.

Applying the APH ECD approach to other clinical disciplines to reduce the burden of documentation for the entire care team is recommended and feasible, based on the results of this project. A practical collaborative opportunity for evaluation of datasets may be to assess satisfaction and burnout metrics published by KLAS Research. ${ }^{17}$ We propose that the implementation of the APH ECD and other strategies to reduce documentation burden before and after could prove beneficial in user satisfaction and burnout measurement. KLAS metrics are widely respected, and increasingly nursing focused.

\section{Conclusion}

Decreasing documentation burden and establishing documentation standards is critical to efficient workflows that support high-quality, safe patient care spanning nursing practice across the globe. These needs highlight the requirement for a standardized approach to nursing documentation. Through the work of this collaborative, a process for identifying essential clinical data elements using evidence-based practice, evidence from nursing practice, and regulatory requirements has been defined and tested by health care systems. This collaborative approach's process and structure are replicable for other venues of care and patient populations.

The high degree of client and vendor partnership and collaboration on the APH ECD is a critical factor in the success of the APH ECD project. Without this partnership, the ability to extract and compare data in standardized formats poses significant challenges given the variation in information system processes between organizations. Identification of data requirements must happen before data elements can be mapped to standard terminologies and shared across platforms.

\section{Clinical Relevance Statement}

Clinical implications of implementing an essential clinical dataset (ECD) within any EMR benefit clinicians, clinical 
informaticians, and the patient. Utilizing the proven methodology illustrated within the study serves informaticians in being able to get to a more efficient way of designing clinical documentation within EMRs. Additionally, the ECD supports clinicians in that there is time saved with a decrease in redundant documentation, and ultimately, our patients benefit, as clinicians will have more time at the bedside to serve them, and therefore affect clinical outcomes.

\section{Multiple Choice Questions}

1. The approach used to define the Essential Clinical Dataset (ECD) was:

a. Consensus-based model, evidence-based practice, and practice exemplars.

b. Evidence-based practice, regulatory and evidence from nursing practice.

c. Data-driven, regulatory and evidence from nursing practice.

d. Regulatory, consensus-based model, and quality framework.

Correct Answer: The correct answer is option b. Defining the ECD required a systematic approach to determining what elements of documentation are required for collection by the Registered Nurse during the admission process. Three areas consistently influenced this practice; evidence-based practice, regulatory requirements, and evidence from nursing practice.

2. Leveraging data collected through the delivery of health care to improve health care is an example of using:

a. Data-driven decision making.

b. Evidence from nursing practice.

c. Evidence-based practice.

d. Consensus driven care.

Correct Answer: The correct answer is option b. Leveraging data gathered through clinically driven and datadriven processes, provides the knowledge and understanding required to identify evidence from nursing practice. Evidence from nursing practice is cited as a vital element of learning health systems, supports workflow efficiencies, unnecessary documentation elements, and improves the overall delivery of health care.

3. Which of the following countries have published position statements for the standardization of nursing assessments:
a. United States of America
b. Canada
c. Australia
d. All of the above

Correct Answer: The correct answer is option d. National nursing organization in the United States, Canada, and Australia have published position papers on the need to standardize nursing assessments to drive quality and safety in the delivery of care. This recognition highlights the importance of defining of an essential clinical dataset. As such, the admission history ECD defined by this collaborative provides foundational work for establishing standardized nursing assessments.

4. The feasibility of the Essential Clinical Dataset demonstrated a reduced burden of documentation via the following results:

a. Reduction in number of clicks.

b. Reduction in time to document the Adult Admission History.

c. Reduction in the total number of questions on the Adult Admission History.

d. All of the above.

Correct Answer: The correct answer is option d. An average reduction in the total number of questions, time, and clicks to complete the APH ECD was the result from early adopter cohorts which is supported by all for the reduction in the burden to nursing documentation.

Protection of Human and Animal Subjects

Human and/or animal subjects were not included in this project.

Conflict of Interest

None declared.

\section{References}

1 Moore EC, Tolley CL, Bates DW, Slight SP. A systematic review of the impact of health information technology on nurses' time. J Am Med Inform Assoc 2020;27(05):798-807

2 O'Brien A, Weaver C, Settergren TT, Hook ML, Ivory CH. EHR documentation: the hype and the hope for improving nursing satisfaction and quality outcomes. Nurs Adm Q 2015;39(04): 333-339

3 Sockolow PS, Liao C, Chittams JL, Bowles KH. Evaluating the impact of electronic health records on nurse clinical process at two community health sites. Ni 2012 (2012) 2012;2012:381

4 Ommaya AK, Cipriano PF, Hoyt DB, Horvath KA, Tang P, Paz HL, DeFrancesco MS, Hingle ST, Butler S, Sinsky CA. 2018. CareCentered Clinical Documentation in the Digital Environment: Solutions to Alleviate Burnout. NAM Perspectives. Discussion Paper. Washington, DC: National Academy of Medicine: 1-10. Doi: $10.31478 / 201801 \mathrm{C}$

5 Collins S, Couture B, Kang MJ, et al. Quantifying and visualizing nursing flowsheet documentation burden in acute and critical care. Paper presented at: AMIA Annual Symposium Proceedings; San Francisco, CA; 2018:348-357

6 ONC, The Office of the National Coordinator for Health Information Technology. 2016 interoperability standards advisory. HealthIT.gov. 2015. Available at: https://www.healthit.gov/sites/ default/files/2016-interoperability-standards-advisory-final-508. pdf. Accessed October 19, 2018

7 Westra BL, Latimer GE, Matney SA, et al. A national action plan for sharable and comparable nursing data to support practice and translational research for transforming health care. J Am Med Inform Assoc 2015;22(03):600-607

8 American Nurses Association. ANA's Principles for Nursing Documentation Guidance for Registered Nurses. MD: Silver Spring; 2010 9 Posner K, Brown GK, Stanley B, et al. The Columbia-Suicide Severity Rating Scale: initial validity and internal consistency findings from three multisite studies with adolescents and adults. Am J Psychiatry 2011;168(12):1266-1277 
10 Karp EL, Freeman R, Simpson KN, Simpson AN. Changes in efficiency and quality of nursing electronic health record documentation after implementation of an admission patient history essential dataset. Comput Inform Nurs 2019;37(05):260-265

11 Wu DTY, Smart N, Ciemins EL, Lanham HJ, Lindberg C, Zheng K. Using EHR audit trail logs to analyze clinical workflow: a case study from community-based ambulatory clinics. AMIA Annu Symp Proc 2018;2017:1820-1827

12 Chen Y, Xie W, Gunter CA, et al. Inferring clinical workflow efficiency via electronic medical record utilization. Paper presented at: AMIA Annual Symposium Proceedings/AMIA Symposium; San Francisco, CA; 2015:416-425

13 Nagle LM, White P. Proceedings from the National Nursing Data Standards Symposium. Canada National Nursing Data Standards Symposium; 2017. Available at: https://cnia.ca/resources/Documents/2017-NNDS-FINAL-Proceedings.pdf. Accessed October 5, 2018
14 Nursing Informatics Position Paper of Australia. 2018. Available at: https://www.hisa.org.au/wp-content/uploads/2017/08/Nursing-Informatics-Position-Statement_06082017.pdf. Accessed November 9, 2018

15 Wilbanks BA, Berner ES, Alexander GL, Azuero A, Patrician PA, Moss JA. The effect of data-entry template design and anesthesia provider workload on documentation accuracy, documentation efficiency, and user-satisfaction. Int J Med Inform 2018; 118:29-35

16 Embi PJ, Payne PRO. Evidence generating medicine: redefining the research-practice relationship to complete the evidence cycle. Med Care 2013;51(08, Suppl 3):S87-S91

17 Interoperabilty 2019: Vendor Success in Making Outside Data Usable. Available at: https://klasresearch.com/report/interoperability-2019/1395. Accessed June 9, 2020 